\title{
Lactational Metformin Treatment in Mouse Dams Ameliorates Maternal High-Fat Diet-induced Metabolic Complications in Male Offspring
}

\author{
Linh V. Nguyen ${ }^{1}$, Khoa D.A Nguyen ${ }^{2}$ and Khanh V. Doan ${ }^{1, *}$ \\ ${ }^{1}$ Department of Pharmacology, ${ }^{2}$ Department of Physiology, Tan Tao University, School of Medicine, Duc Hoa, Long An 850000, Viet Nam, ${ }^{*}$ Corresponding \\ authors: E-mail: khanh.doan@ttu.edu.vn
}

\begin{abstract}
Maternal obesity/diabetes severely affects the offspring's metabolism not only during the fetal and early life development but also later on in the childhood and adulthood stages. Recent evidence suggested that maternal metformin treatment during pregnancy and breastfeeding might provide long-term metabolic benefits on offspring's glucose homeostasis. We employed a mouse model of maternal overnutrition induced by a high-fat diet (HFD) to investigate whether an early metformin treatment in mouse dams during the lactation period attenuates the maternal HFD-induced metabolic complications in male offspring. The results showed that male offspring from metformin-exposed mothers during the suckling period displayed lower body weight and decreased white fat contents. Furthermore, these male offspring had lower blood glucose levels, decreased hyperinsulinemia, enhanced glucose tolerance and insulin sensitivity. Moreover, we found that short-term metformin treatment during the lactation period in mouse dams solely improved the glucose tolerance of male offspring exposed to high-fat diet (HFD) during both in utero and early postnatal stages, which might primarily result from an increase in insulin secretion. However, this metformin treatment in mouse dams was enabled to abolish most of the metabolic complications of the male offspring exposed to HFD during the lactation period. Together, these findings suggest that early intervention in mouse dams by metformin treatment during the lactation period may provide long-term metabolic benefits in the regulation of male offspring's glucose homeostasis.
\end{abstract}

\section{KEYWORDS \\ Maternal obesity, Lactation, Metformin, Offspring, Metabolic disorders}

\section{ARTICLE HISTORY}

Received: 15.08 .2020

Accepted: 20.09 .2020

Online: 01.10 .2020

DOI: https://doi.org/10.53901/tzug7sy729f

\section{INTRODUCTION}

Obesity among children, adolescents, and adults associating with many related psychosocial and cardiometabolic disorders at later age such as hypertension, dyslipidemia, and insulin resistance have become one of the most serious public health issues nowadays [1]. Maternal obesity or high-fat diet exposure, a realistic situation that has become a worldwide epidemic, might be one of the important factors contributing to this increasing rate of childhood obesity. Enormous epidemiological evidence has shown that maternal obesity has many adverse effects on fetal develop-

Dedicated the 10th Anniversary of Tan Tao University ment conditioning offspring for life-long risk of cardiometabolic diseases [2]. Early effective interventions to reduce offspring's metabolic disorders in a later stage of life should be incorporated in a comprehensive management approach to childhood obesity.

The gestational period is indisputably critical for the fetal developmental programming of offspring and has been the focus of much previous work. However, the early postnatal period development is also an important time for the continuing establishment of body composition, plasticity of metabolic organs, beta cell mass, and insulin sensitivity. Evidence from animal models of maternal high fat diet exposure suggested that this lactation period might 
have an independent but equally important programming effect on the offspring's metabolic health [3]. Indeed, lactational overnutrition adversely affects the adult offspring's metabolic health, increasing fat mass, impairing brown fat thermogenic function, and increased susceptibility to diet-induced obesity in adulthood $[4,5]$. The lactation period may therefore offer a window of opportunity for an early intervention to improve offspring's glucose homeostasis [6].

Metformin, a biguanide agent, is considered the first-line therapeutic medication in the prevention and/or treatment of type 2 diabetes basing on vast data on its efficacy and safety [7, 8]. Metformin is generally considered an alternative therapeutic option in the treatment of gestational diabetes when insulin, the gold standard treatment, is not suitable [9]. Due to its ability to cross the placenta and limited long-term safety data on offspring, clinical use of metformin in the treatment of gestational has been concerned $[10,11]$. However, there is accumulating evidence both in animals and humans indicated that metformin is an effective and safe option for the treatment of diabetes during pregnancy and breastfeeding, providing some metabolic benefits on offspring compared to insulin without adverse effects on long-term growth and development $[12,13]$. However, most of these animal studies investigated the effects of maternal metformin exposure throughout both gestation and lactation periods or just focusing on the gestation period. There is still a very limited number of studies investigating the effects of maternal metformin treatment during lactation on offspring's metabolic health.

In this study, we investigated whether short-term metformin exposure during the suckling period attenuates the maternal highfat diet-induced metabolic complications in murine offspring. We found that lactational metformin treatment improved glycemic control without affecting the bodyweight of the mouse dams. Male offspring from metformin-exposed mothers during lactation displayed lower postnatal and/or post-weaning body weight and decreased white fat contents. Moreover, these male offspring had improved glucose homeostasis including lower blood glucose levels, decreased hyperinsulinemia, enhanced glucose tolerance, and insulin sensitivity. Furthermore, we found that in the male offspring exposed to a high-fat diet (HFD) during both in utero and early postnatal stage, short-term metformin treatment in mouse dams during the lactation period solely improved the offspring's glucose tolerance, which might primarily result from an increase in insulin secretion. However, this metformin treatment in mouse dams was enabled to abolish most of the metabolic complications of the male offspring exposed to HFD during the lactation period, which might be due to multiple effects on the offspring's adipogenesis and insulin sensitivity. Together, these findings suggest that early intervention in mouse dams by metformin treatment during the lactation period may provide long-term metabolic benefits to maintain glucose homeostasis in male offspring.

\section{MATERIALS AND METHODS}

\section{Animals}

All animal experiments in this study were approved by the Institutional Animal Care and Use Committee (IACUC) of the Tan Tao University, School of Medicine (Certificate No. 03/2018HDKH.TTU). Four to six-week-old SWISS mice were purchased from Pasteur Institute (Ho Chi Minh city, Viet Nam). Mice were kept in controlled room temperature $\left(22 \pm 1^{\circ} \mathrm{C}\right)$ with a 12-hourlight/dark cycle (light on/off at 06:00 a.m./p.m.). Mice were fed a normal chow diet (AniFood, Pasteur Institute-VN, $3.84 \mathrm{kcal} / \mathrm{kg}$

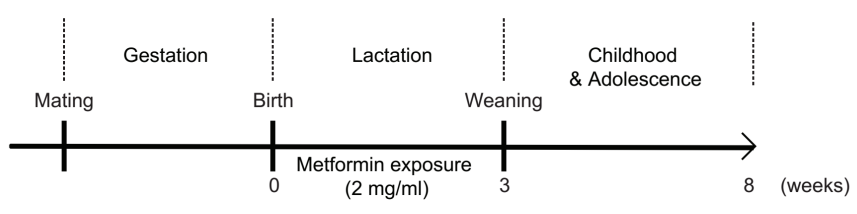

\begin{tabular}{|l|l|l|}
\hline \multicolumn{2}{|c|}{ Diet and Treatment of Dams } & Groups \\
\hline Normal chow (NC) & Normal chow (NC) & Control (Ctr) \\
\hline Normal chow (NC) & High fat diet (HF) & LacHF \\
\hline High fat diet (HF) & High fat diet (HF) & GesLacHF \\
\hline Normal chow (NC) & HF + Metformin & LacHF-Met \\
\hline High fat diet (HF) & HF + Metformin & GesLacHF-Met \\
\hline
\end{tabular}

Figure 1 Scheme of experimental design.

with $6-8 \% \mathrm{kcal}$ from fat, NC) with filtered water provided ad libitum.

Only female mice which had one litter previously were used to cross with SWISS males for experiments in this study. All female dams were fed either NC diet or high fat diet (Research Diets D12492, USA, $5.24 \mathrm{kcal} / \mathrm{kg}$ with $60 \% \mathrm{kcal}$ from fat, HF) on the first day of mating and maintained this diet throughout the gestation period. Male mice were removed from the breeding cages after confirmation of the mating success by checking the presence of vaginal plug in females. On the day of birth, the female dams which were fed with $\mathrm{NC}$ during gestation were randomly divided into three groups: one continued to be fed with NC diet and filtered water ad libitum serving as control group; one was switched to HF diet and received filtered water as vehicle treatment; and the other was switched to HF diet and received metformin treatment (groups Ctr, LacHF and LacHF-Met, Figure 1). Dams which were fed with HF diet during gestation were randomly split into two groups: one continued with HF diet and received filtered water as vehicle treatment; and the other continued with HF diet and received metformin treatment (groups GesLacHF and GesLacHFMet, Figure 1). To avoid bias in litter size, maternal pup care, and quantity of milk received, only dams having litters of 8-12 pups were selected and litters were standardized to ten pups per litter at birth.

Dams received metformin (TOKU-E, Bellingham, WA, USA) via drinking water $(2 \mathrm{mg} / \mathrm{ml})$ throughout a 3-week lactation period (16). At weaning time, the metformin treatment was discontinued and the offspring from all groups were fed with a NC diet. After weaning, only male offspring were used to monitor and evaluated for the metabolic parameters. Experiments were repeated at least twice to ensure reproducibility of the detected phenotypes.

\section{Body weight monitoring}

The body weight and blood glucose levels of mouse dams were monitored weekly from mating time to weaning day. Offspring's body weight was monitored weekly from delivery day to weaning time. After weaning, male offspring continued to have their body weights traced weekly for 5 more weeks (until reaching 8year-old). At this time, male offspring were divided into several subsets to evaluate multiple metabolic parameters including food intake, body length and white fat contents, blood glucose levels, 
glucose and insulin tolerance tests, and in vivo glucose-stimulated insulin secretion test. After the experimental period, mice were sacrificed by cervical dislocation after anesthetizing with ketamine (100 mg/ kg body weight) and the organs and plasma samples were collected and stored at $-80 \mathrm{oC}$ for further analyses.

\section{Blood glucose measurement}

Blood glucose level was determined from the blood drop taken from a tail nick by the glucose oxidase method using a commercial blood glucometer (SAFE-ACCU, Shanghai International Holding Corp., Germany). For fed state blood glucose, mice were removed from food 2 hours before measurement. To determine fasted state blood glucose, mice were fasted overnight (16 hours) with water provided ad libitum.

\section{Glucose and insulin tolerance tests}

We performed glucose and insulin tolerance tests (GTT and ITT) when the offspring was at 8 weeks old as described previously (17). Briefly, mice were fasted for 16 hours or 2 hours before GTT and ITT testing, respectively. Mice were then administered intraperitoneally (i.p.) with $1.5 \mathrm{~g} / \mathrm{kg}$ D-glucose or $0.5 \mathrm{U} / \mathrm{kg}$ insulin (Humulin ${ }^{\circledR}$ U-100, Eli Lilly and Co., IN, USA) prepared in normal saline for GTT and ITT, respectively. Blood samples were taken from a tail nick at $0,20,40,60,80,120$, and 160 minutes after injection and glucose levels were measured by glucose oxidase method using a glucometer. The area under the curve (AUC) was determined to quantify the glucose and insulin tolerance.

During the GTT test, blood samples were also collected before glucose administration, and at 40 and 80 minutes after injection to analyze plasma insulin levels in order to evaluate the in vivo glucose stimulated insulin secretion (GSIS) of mice.

\section{Blood collection and plasma sample preparation}

Blood samples were gently collected in EDTA-coated tubes (Microvette ${ }^{\circledR}$ CB 300, Sarstedt, Germany) via a tail nick and centrifuged at 10,000 rpm, $4 \mathrm{oC}$ for 10 minutes. The plasma supernatants were collected and stored at $-80 \mathrm{oC}$ before analyzed.

\section{Leptin and insulin measurement}

Plasma insulin and leptin levels were determined using ELISA kits (Cat. No. 90080, Crystal Chem, USA, for insulin and Cat. No. MBS761657, MIoBS, Japan, for leptin) in accordance with manufacturer's instructions.

\section{Western blotting}

Antibodies used for immunoblotting include AMPK (Cell Signaling, Cat. No. 2532, 1:5,000), p-AMPK (Cell Signaling, Cat. No. 2535, 1:5,000), GAPDH (GeneTex, Cat. No. GTX100118, 1:10,000) and anti-Prolactin (Santa Cruz, Cat.No. sc-7805, 1:500). The primary antibodies were prepared in 3\% bovine albumin in Tris-buffered saline containing $0.1 \%(\mathrm{v} / \mathrm{v})$ Tween 20 (TBST) and $0.05 \%(\mathrm{~m} / \mathrm{v})$ sodium azide with dilution factors as indicated. The anti-goat and anti-rabbit secondary antibodies conjugated with horseradish peroxidase (HRP) were purchased from Thermo Scientific (Thermo Fisher Scientific Inc., MA, USA) and were diluted 10,000 times in $3 \%$ non-fat dry milk dissolved in TBST. All other reagents were purchased from Sigma-Aldrich unless otherwise stated.

Tissues were homogenized with a glass Dounce homogenizer and lysed with RIPA buffer containing protease (Thermo Fisher Scientific Inc., MA, USA) and phosphatase (F. Hoffmann-La Roche Ltd., Basel, Switzerland) inhibitors. PierceTM Coomassive (Bradford) Protein Assay Kit (Thermo Fisher Scientific Inc., MA,
USA) was used to measure the total protein concentrations in lysed or plasma samples. $20 \mu \mathrm{g}$ of the total protein in lysates or plasma samples were run on SDS-PAGE gel and transferred onto nitrocellulose membranes. After blocking with 5\% non-fat dry milk dissolved in TBST for an hour, the membranes were probed with a given primary antibody overnight at $4^{\circ} \mathrm{C}$, reacted with HRP-conjugated anti-rabbit or anti-goat IgG secondary antibodies. After washing out for the unbound antibodies, membranes were exposed to the X-ray film (UltraCruz ${ }^{\circledR}$ Autoradiography Film, Santa Cruz Biotechnology Inc., TX, USA) using the SuperSignal ${ }^{\mathrm{TM}}$ West Femto Maximum Sensitivity Substrate (Thermo Fisher Scientific Inc., MA, USA). NIH ImageJ software was used to perform the densitometry of film blots.

\section{STATISTICS}

The data are represented as mean \pm SEM. Statistical significance was determined by 2-tailed Student's t-test, one- or two-way analysis of variance (ANOVA). GraphPad PRISM version 5.0 was used for the statistical analyses, and p-value less than 0.05 was considered as a statistically significant difference.

\section{RESULTS}

\section{Metformin treatment during lactation improved glycemic control in mouse dams}

The experimental procedures are summarized in Figure 1. Body weight of mouse dams did not change between dams fed with NC diet (Ctr group), dams fed with HFD during the gestation and lactation treated with vehicle (HF group) or metformin (HF-Met group) (Figure 2A). After 2 weeks of metformin treatment, dams exposed to metformin showed lower blood glucose and plasma insulin levels, indicating a better glycemic control in the dams treated with metformin (Figure 2B and Figure 2C). The effect of metformin treatment on blood glucose and plasma insulin was accompanied by an enhancement of in vivo AMPK activation, which is a well-established mechanism of action of metformin (Figure 2D). In addition, plasma prolactin, an important hormone regulating milk production and secretion, did not differ between dams treated with vehicle or metformin during suckling period, though we observed a decreasing tendency of plasma prolactin in dams fed with HFD compared to dams fed with NC (Figure 2E). These results suggested that treatment with metformin during lactation period improved blood glucose control without altering the body weight and suckling ability of mouse dams.

\section{Male offspring from mouse dams exposed to metformin during lactation exerted anti-obesity phenotypes}

We next monitored pup phenotypes from delivery day to weaning time to access offspring's early postnatal development. Pup body weight was not different between groups at birth (data not shown). However, one week postnatal, pups from mothers exposed to HFD during lactation and gestation period (LacHF and GesLacHF) had higher body weights compared to those from mothers of Ctr group (Figure 3A, left panel). We noticed that body weight of pups from dams exposed to HFD during both gestation and lactation periods (GesLacHF) was slightly, but significantly, higher than that of pups from dams exposed to HFD during lactation period only (LacHF) (Figure 3A, left panel). Interestingly, early metformin exposure during lactation in mouse dams completely abolished the increase in body weight of pups from LacHF dams but not that of pups from GesLacHF mothers (Figure 3A, left panel). The 
A,

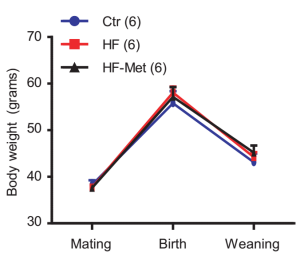

B,

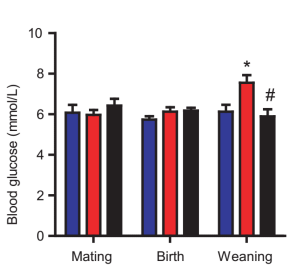

E,

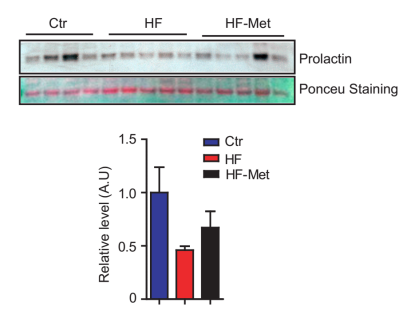

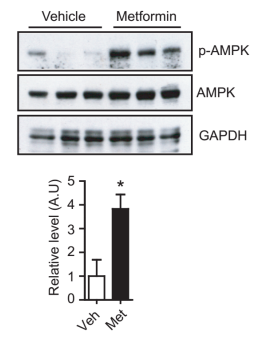

c,
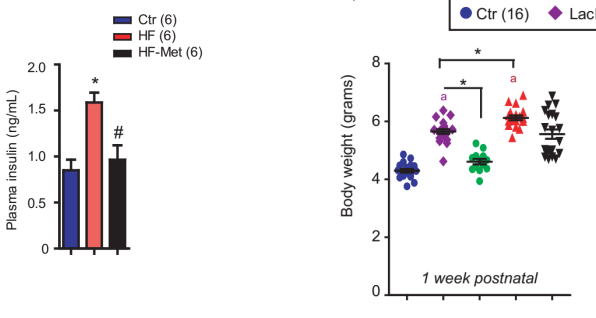

B,

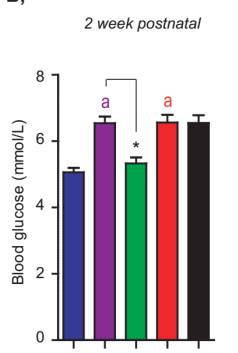

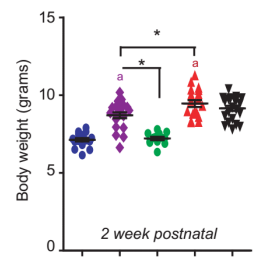

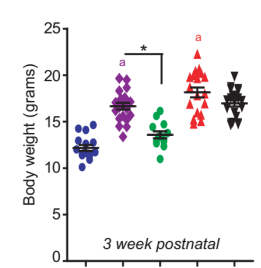

C,

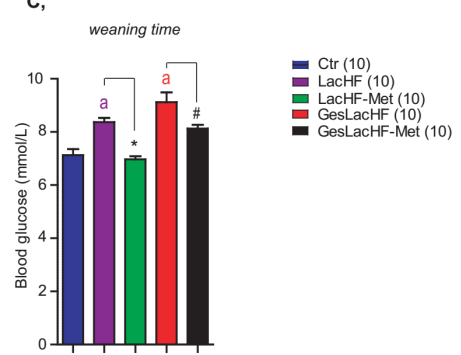

Figure 2 Improved glycemic control in mouse dams treated with metformin during lactation period.

A, Body weight of control dams (Ctr) and dams fed with HFD treated with vehicle (HF) or metformin (HF-Met). B, C, Blood glucose levels in fed state (B) and plasma insulin of control dams (Ctr) and dams fed HFD treated with vehicle (HF) or metformin (HF-Met). D, Immunoblots (top) and graph (bottom) showing levels of $\mathrm{p}$-AMPK in the livers of dams treated with vehicle (Veh) or metformin (Met). E, Immunoblots (top) and graph (bottom) showing plasma prolactin levels of control dams (Ctr) and dams fed HFD treated with vehicle (HF) or metformin (HF-Met). Data are presented as mean \pm SEM. Two-way ANOVA in line graph and one-way ANOVA with Turkey's post-tests or Student's t-tests in bar graphs. ${ }^{*} P<0.05$ compared to Ctr group, $\# P<0.05$ compared to HF group.

aforementioned differences in body weight of these groups of mice offspring was consistently observed until weaning time (Figure $3 \mathrm{~A}$, middle and right). Furthermore, we found that pups from dams treated with metformin during lactation (LacHF-Met and GesLacHF-Met) had lower blood glucose compared to those from LacHF and GesLacHF mothers at the second week postnatal or at weaning time (Figure 3B and (Figure 3C). These results highlighted early metabolic changes induced by maternal HFD challenge.

We continued to track the male offspring's body weight after weaning the pups onto a NC diet until they reached the adolescent age of 8-week-old. The same body weight phenotypes of these male offspring were recorded during post-weaning development (Figure 4A) though they consumed comparable food intake (Figure 4B). Higher white fat contents might primarily contribute to the heavier body weight phenotype of male offspring exposed to maternal HFD compared to control offspring as their body lengths were not different (Figure 4C and Figure 4D). Unexpectedly, male offspring from LacHF dams had lower content of brown adipose tissue (BAT) compared to control offspring (Figure 4D). Strikingly, male offspring from LacHF-Met dams displayed a significant decrease in epidydimal white adipose tissues (eWAT) and restored BAT content compared to those from LacHF group (Figure 4D). Compared to the male offspring from GesLacHF group, the lactational metformin treatment significantly reduced the inguinal white fat adipose tissue (iWAT) content (Figure 4D). These results
Figure 3 Early metabolic changes induced by maternal HFD feeding were attenuated by lactational metformin treatment. A, Postnatal body weight of male offspring until weaning time. B, C, Blood glucose level of male offspring at 2-week postnatal (B) and at weaning time $(C)$. Data are presented as mean \pm SEM.

One-way ANOVA with Turkey's post-tests. a $P<0.001$ compared to $\mathrm{Ctr}$ group, ${ }^{*} P<0.05$ compared to LacHF group, $\# P<0.05$ compared to GesLacHF group.

indicated that short-term metformin treatment in mouse dams during lactation period reduced risk of obesity development in male offspring.

Metformin treatment in mouse dams during lactation improved glucose homeostasis of male offspring in later life stage

We further investigated metabolic parameters of male offspring after weaning to evaluate their glucose homeostasis in later life stage. Male offspring from LacHF and GesLacHF dams displayed a hyperglycemic condition as their blood glucose levels both in normal (fed) and fasted state were much higher than those of control offspring (Figure 5A). Moreover, these male offspring had significant increases in plasma insulin and leptin levels compared to control offspring (Figure 5B and Figure 5C). The hyperglycemic condition together with hyperinsulinemia and hyperleptinemia indicated a dysregulation of the glucose homeostasis in these male offspring. Interestingly, while lactational metformin treatment in mouse dams helped to correct all the phenotypes of increased blood glucose, insulin and leptin levels of male offspring from LacHF dams, this metformin treatment only reduced the hyperglycemia, but not the hyperinsulinemia and hyperleptinemia, of the male offspring from GesLacHF dams (Figure 5A-C). These results implicated that short-term dam treatment with metformin during lactation might improve the glucose homeostasis of male offspring in a different manner.

We next therefore performed glucose and insulin tolerance tests (GTT and ITT) to further figure out the beneficial effects of lactational metformin treatment on male offspring's glucose homeostasis. Male offspring from both LacHF-Met and GesLacHF-Met dams showed higher ability to tolerate an exogenous glucose loading compared to those mice from dams untreated with metformin 
A,

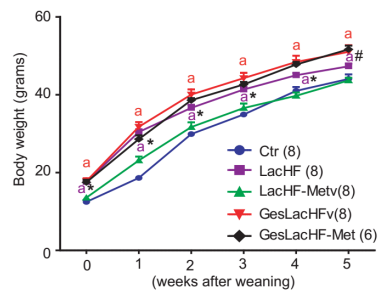

C,

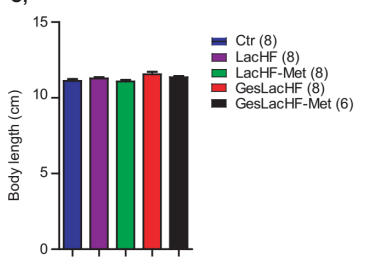

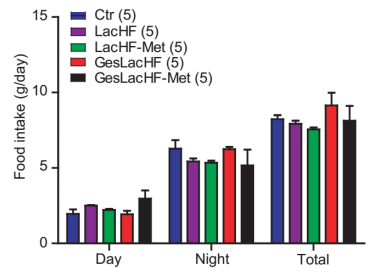

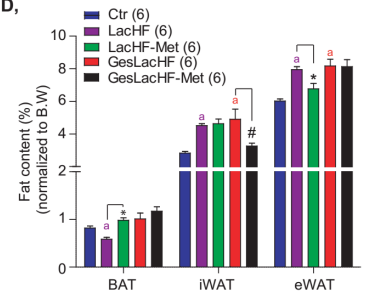

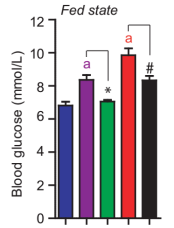

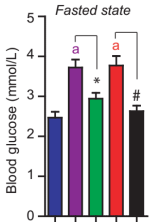

D,

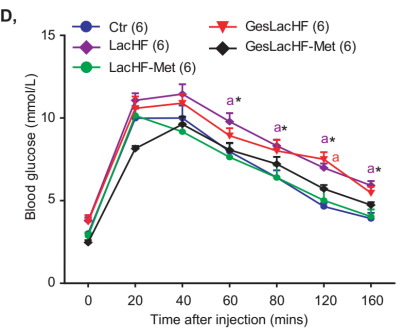

$\mathrm{F}$,
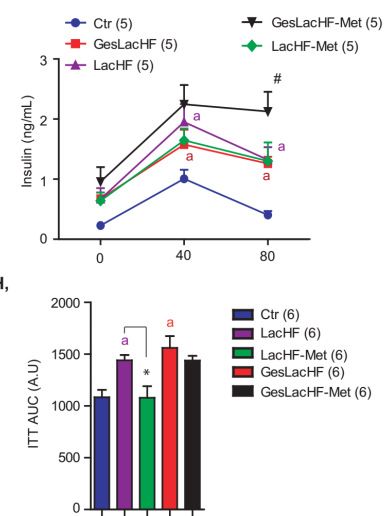

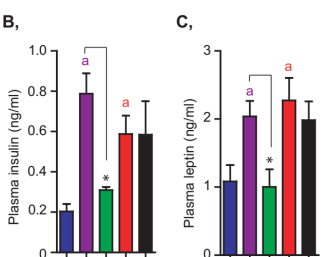

$E$,

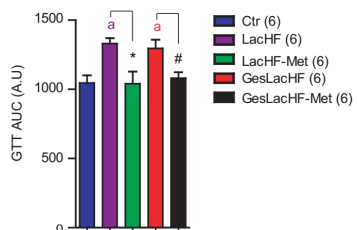

G,

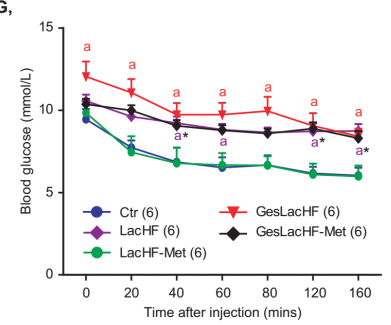

Bonferroni's post-tests in line graph a $P<0.001$ compared to $C$ tr group, ${ }^{*} P<0.05$ compared to LacHF group, $\# P<0.05$ compared to GesLacHF group.

(Figure 5D and Figure 5E). We analyzed insulin levels during the GTT testing to access ability of glucose-responsive insulin secretion and found that male offspring from GesLacHF-Met dams, but not those from LacHF-Met dams, displayed a significant increase in the in vivo glucose-stimulated insulin secretion (GSIS) compared to male offspring from dams untreated with metformin (Figure 5F). In contrast, the ITT test revealed that male offspring from LacHF-Met dams, but not those from GesLacHF-Met dams, exhibited a significant improvement of insulin sensitivity compared to mice offspring from vehicle-treated dams (Figure 5G and Figure $5 \mathrm{H}$ ). These results indicated that short-term lactational metformin treatment might help to improve male offspring's glucose homeostasis by increasing pancreatic beta-cell insulin secretion and/or by enhancing insulin sensitivity depending on timeframe of the maternal HFD exposure.

\section{DISCUSSION}

Accumulating evidence from both animal and human studies suggests beneficial effects of maternal metformin treatment on offspring's metabolism. Most of these studies focused on the maternal metformin exposure during gestation or throughout both gestation and lactation periods [13]. There is a very limited number of studies examining the individual effect of metformin treatment during lactation period on offspring's metabolic health, even though recent evidence indicated that this early postnatal period is crucial for integral development of the offspring's metabolic organs and functions in order to maintain their glucose homeostasis in later life.

In our study, mouse dams fed HFD during lactation only or both gestation and lactation periods provoked early and long-term consequences on the offspring's metabolic phenotype. These adverse effects of maternal HFD exposure included an early increase

Figure 5 Lactational metformin treatment in dams improved glucose homeostasis of male offspring.

A, Blood glucose levels in fed (left) and fasted (right) states of male offspring. B, C, Plasma insulin (B) and leptin (C) levels of male offspring. D, E, GTT (D) and GTT AUC (E) of male offspring. F, In vivo glucose-stimulated insulin secretion in male offspring. $G, H$, GTT $(\mathrm{G})$ and GTT AUC $(\mathrm{H})$ of male offspring. Data are presented as mean \pm SEM. One-way ANOVA with Turkey's post-tests in bar graphs and two-way ANOVA with Bonferroni's post-tests in line graph. a $P<0.01$ compared to $C$ tr group, ${ }^{*} P<0.05$ compared to

LacHF group, $\# P<0.05$ compared to GesLacHF group.

in body weight and blood glucose, increased adiposity, and dysregulated glucose homeostasis in the male offspring in later life. Our findings were consistent with previous studies indicating that maternal HFD exposure during lactation only seemed to have comparable and independent effects on male offspring's adiposity and glucose homeostasis compared to maternal HFD exposure during both gestation and lactation periods, even though offspring from dams exposed to HFD during both gestation and lactation exerted slightly higher body weight. For example, Masuyama and colleagues showed that maternal HFD during suckling had an additive effect on the offspring's metabolic disorder irrespective of the nutritional status in utero [5]. Furthermore, Sun et al. suggested that maternal HFD during lactation had an even greater influence on offspring's metabolic phenotype than gestational HFD diet exposure [14].

Remarkably, we found that short-term metformin treatment in mouse dams during lactation could improve the glucose homeosta- 
sis of male offspring. In male offspring of dams exposed to HFD during both gestation and lactation, improved glucose homeostasis by metformin treatment might be primarily resulted from the increased pancreatic insulin secretion in addition to the reduced iWAT content. This finding in our study is in agreement with the results of a recent study in which Gregg and colleagues reported that maternal exposure to metformin improved male mouse offspring's glucose tolerance and insulin secretion [15]. In male offspring of dams exposed to HFD during lactation, metformin treatment in mouse dams might promote the offspring's glucose homeostasis by different manners attributed to the improvements in body weight, eWAT content, plasma metabolic hormones, and insulin sensitivity. Moreover, the ability to restore reduced BAT content in male offspring from dams fed HFD during lactation might also contribute to the beneficial action of lactational metformin treatment on offspring's glucose homeostasis as a recent study also reported that impaired BAT function due to maternal HFD during lactation resulted in the deterioration of offspring's glucose homeostasis which could be attenuated by early postnatal metformin administration [16]. These multiple benefits may provide an explanation for our observation that lactational metformin treatment completely abolished most of the metabolic complications including the hyperglycemia, hyperinsulinemia and hyperleptinemia induced by maternal HFD exposure during lactation in male mice offspring.

There are a few limitations in our current study regarding undefined mechanisms underlying the effect of lactational metformin treatment in mouse dams on the offspring. For example, because the presence of metformin in plasma and breast milk of mouse dams and in offspring's plasma were not measured in our study, we therefore could not rule out the possibility that there was an amount of metformin secreted in breast milk from dam's plasma. Such an amount of metformin in ingested milk could be absorbed into the offspring's circulating system and resulted in direct effects on offspring's body tissues. However, studies in humans demonstrated that though metformin was transferred into breast milk, the concentration of metformin in breast milk was generally low and did not affect blood glucose of infants [17, 18]. Moreover, in a recently published study using a similar scheme of lactational metformin treatment with higher dose of metformin compared to our study, Zach and colleagues found that the metformin levels in offspring's plasma were very low or undetectable [19]. We therefore postulated that a direct effect of metformin levels in offspring's blood in our study should be minor and unlikely to contribute to the effects of lactational metformin treatment on offspring's metabolic phenotypes observed in our study.

Another possible mechanism might relate to alterations in maternal metabolic state occurred during lactation period caused by metformin treatment, which may influence milk quantity and composition which have not been determined in the current study. Although there is no study in rodents investigating the effect of maternal metformin treatment on breastfeeding ability, evidence in humans suggested that metformin had no impact on breast size and breastfeeding [20]. As lactational metformin treatment did not alter either maternal body weight or prolactin levels in the current study, we may assume that milk quantity might be not affected by this metformin treatment. Because maternal obesity and high fat feeding strongly influenced both milk macro- and micro-nutrient composition [21, 22] and there was no study reporting an effect of maternal metformin on milk composition, effect of maternal metformin on milk composition could be possible and warrant future study. Moreover, maternal obesity and high fat feeding have been shown to associate with the alterations in gut microbiome of offspring which has recently been suggested to modulate weight gain, alter metabolism, and drive obesity and cardiometabolic diseases $[2,23,24]$. Thus, whether maternal metformin treatment can result in a maternal-fetal-infant microbial shift relationship will be a very interesting area for future studies.

\section{CONCLUSION}

In the current study we have shown that an early metformin treatment in mouse dams during lactation period resulted in longterm metabolic benefits in maintenance of male offspring's glucose homeostasis. The beneficial effects of lactational metformin treatment might be due to multiple effects on offspring's adipogenesis, insulin secretion and insulin sensitivity depending on the timeframe of maternal HFD exposure. Future studies are required to understand how this maternal metformin treatment influences milk composition and infant gut microbiome to induce these metabolic benefits.

\section{AUTHOR CONTRIBUTIONS}

Linh Nguyen performed experiments and analyzed data. Khoa Nguyen analyzed data and draft manuscript. Khanh Doan conceptualized the research, analyzed data, wrote, edited and finalized the manuscript.

\section{CONFLICT OF INTEREST}

The authors declare no competing interests.

\section{ACKNOWLEDGEMENTS}

This work was supported by the Tan Tao University (TTU) Foundation for Science and Technology Development under grant number TTU.RS.19.305.025. We sincerely thank Professor Ki Woo Kim (Department of Oral Biology, Yonsei University-School of Dentistry, Seoul, Republic of Korea) for his generous providing of primary antibodies used in this study.

\section{REFERENCES}

[1] Neslihan Güngör. Overweight and obesity in children and adolescents. Journal of clinical research in pediatric endocrinology, 6:129-43, 092014.

[2] Caterina Neri and Andrea Edlow. Effects of maternal obesity on fetal programming: Molecular approaches. Cold Spring Harbor perspectives in medicine, 6, 092015.

[3] G. A. Ribaroff, E. Wastnedge, A. J. Drake, R. M. Sharpe, and T. J. G. Chambers. Animal models of maternal high fat diet exposure and effects on metabolism in offspring: a metaregression analysis. Obesity reviews : an official journal of the International Association for the Study of Obesity, 18(6):673-686, Jun 2017. 28371083[pmid].

[4] Tanyara Baliani Payolla, Simone Ferreira Lemes, Thaís de Fante, Andressa Reginato, Cristiano Mendes da Silva, Thayana de Oliveira Micheletti, Hosana Gomes Rodrigues, Adriana Souza Torsoni, Marciane Milanski, and Marcio Alberto Torsoni. High-fat diet during pregnancy and lactation impairs the cholinergic anti-inflammatory pathway in the liver and white adipose tissue of mouse offspring. Molecular and cellular endocrinology, 422:192-202, February 2016.

[5] Hisashi Masuyama and Yuji Hiramatsu. Additive effects of maternal high fat diet during lactation on mouse offspring. PloS one, 9:e92805, 032014. 
[6] Lindsay Ellsworth, Emma Harman, Vasantha Padmanabhan, and Brigid Gregg. Lactational programming of glucose homeostasis: a window of opportunity. Reproduction (Cambridge, England), 156(2):R23-R42, Aug 2018. 29752297[pmid].

[7] Elizabeth Sanchez-Rangel and Silvio E Inzucchi. Metformin: clinical use in type 2 diabetes. Diabetologia, 60(9):1586-1593, September 2017.

[8] Vanita R. Aroda, William C. Knowler, Jill P. Crandall, Leigh Perreault, Sharon L. Edelstein, Susan L. Jeffries, Mark E. Molitch, Xavier Pi-Sunyer, Christine Darwin, Brandy M. Heckman-Stoddard, Marinella Temprosa, Steven E. Kahn, David M. Nathan, and Diabetes Prevention Program Research Group. Metformin for diabetes prevention: insights gained from the diabetes prevention program/diabetes prevention program outcomes study. Diabetologia, 60(9):1601-1611, Sep 2017. 28770322[pmid].

[9] Kristi Kelley, Dana Carroll, and Allison Meyer. A review of current treatment strategies for gestational diabetes mellitus. Drugs in context, 4:212282, 072015.

[10] Jane Tarry-Adkins, Catherine Aiken, and Susan Ozanne. Neonatal, infant, and childhood growth following metformin versus insulin treatment for gestational diabetes: A systematic review and meta-analysis. PLOS Medicine, 16:e1002848, 082019.

[11] Linda A. Barbour and Denice S. Feig. Metformin for gestational diabetes mellitus: Progeny, perspective, and a personalized approach. Diabetes Care, 42(3):396-399, 2019.

[12] Suzanne N. Landi, Sarah Radke, Kim Boggess, Stephanie M. Engel, Til Stürmer, Anna S. Howe, and Michele Jonsson Funk. Comparative effectiveness of metformin versus insulin for gestational diabetes in new zealand. Pharmacoepidemiology and Drug Safety, 28(12):1609-1619, 2019.

[13] Gonzalo Jorquera, Bárbara Echiburú, Nicolás Crisosto, Ramón Sotomayor-Zárate, Manuel Maliqueo, and Gonzalo Cruz. Metformin during pregnancy: Effects on offspring development and metabolic function. Frontiers in Pharmacology, 11:653, 062020.

[14] Bo Sun, Ryan Purcell, Chantelle Terrillion, Jianqun Yan, Timothy Moran, and Kellie Tamashiro. Maternal high-fat diet during gestation or suckling differentially affects offspring leptin sensitivity and obesity. Diabetes, 61:2833-41, 062012.

[15] Brigid Gregg, Nathalie Botezatu, Joshua Brill, Hannah Hafner, Suryakiran Vadrevu, Leslie Satin, Emilyn Alejandro, and Ernesto Bernal-Mizrachi. Gestational exposure to metformin programs improved glucose tolerance and insulin secretion in adult male mouse offspring. Scientific Reports, 8, 122018.

[16] Xingwei Liang, Qiyuan Yang, Lupei Zhang, Joseph Maricelli, Buel Rodgers, and Meijun Zhu. Maternal high-fat diet during lactation impairs thermogenic function of brown adipose tissue in offspring mice. Scientific reports, 6:34345, 092016

[17] Thomas Hale, J Kristensen, L Hackett, Rolland Kohan, and Kenneth Ilett. Transfer of metformin into human milk. Diabetologia, 45:1509-14, 122002.

[18] Gerald G Briggs, Peter J Ambrose, Michael P Nageotte, Guadalupe Padilla, and Stephanie Wan. Excretion of metformin into breast milk and the effect on nursing infants. Obstetrics and gynecology, 105(6):1437-1441, June 2005.

[19] Zach Carlson, Hannah Hafner, Molly Mulcahy, Kaylie Bullock, Allen Zhu, Dave Bridges, Ernesto Bernal-Mizrachi, and Brigid Gregg. Lactational metformin exposure programs offspring white adipose tissue glucose homeostasis and resilience to metabolic stress in a sex-dependent manner. American Journal of Physiology-Endocrinology and Metabolism, 318(5):E600-E612, 2020. PMID: 32154743.

[20] Eszter Vanky, Jj Nordskar, H Leithe, Ak Hjorth-Hansen, Marit Martinussen, and Sven Carlsen. Breast size increment during pregnancy and breastfeeding in mothers with polycystic ovary syndrome: A follow-up study of a randomised controlled trial on metformin versus placebo. BJOG : an international journal of obstetrics and gynaecology, 119:1403-1409, 07 2012.

[21] Monika Słupecka-Ziemilska, Katarzyna Romanowicz, and Jarosław Woliński. Maternal high-fat diet during pregnancy and lactation influences obestatin and ghrelin concentrations in milk and plasma of wistar rat dams and their offspring International Journal of Endocrinology, 2016:1-9, 032016.

[22] Gabriela E. Leghi, Merryn J. Netting, Philippa F. Middleton, Mary E. Wlodek, Donna T. Geddes, Muhlhausler, and Beverly S. The impact of maternal obesity on human milk macronutrient composition: A systematic review and meta-analysis. Nutrients, 12(4):934, Mar 2020. 32230952[pmid].

[23] Jeffrey Galley, Michael Bailey, Claire Kamp Dush, Sarah Schoppe-Sullivan, and Lisa Christian. Maternal obesity is associated with alterations in the gut microbiome in toddlers. PloS one, 9:e113026, 092014.

[24] Derrick Chu, Kathleen Antony, Jun Ma, Amanda Prince, Lori Showalter, Michelle Moller, and Kjersti Aagaard. The early infant gut microbiome varies in association with a maternal high-fat diet. Genome Medicine, 8, 122016. 